\title{
Study on Characteristics of Magnetic Memory Signal of Q345R Steel during the Tensile Process
}

\author{
Chao Yang ${ }^{1, *}$, Yiliang Zhang ${ }^{1}$ and Xiaoyang $\mathrm{Li}^{1}$ \\ ${ }^{1}$ College of Mechanical Engineering and Applied Electronics Technology, Beijing University of \\ Technology, Beijing, China, 100124
}

${ }^{*}$ Corresponding author: yangchao2014@emails.bjut.edu.cn

Keywords: magnetic memory, tensile process, initial yielding, mean local derivative.

\begin{abstract}
A systematic experiment research is carried out on the study of magnetic memory, aiming at the problem of quantitative relationship between the strain varying process and the magnetic memory signals. The Q345R steel was used as the object material. A large plate specimen was used to perform the magnetic memory test at typical deformation stages of the stretching process. In this paper, a mean local derivative $(\overline{\mathrm{dH} / \mathrm{dx}})$ method is put forward in the mathematical characterization of magnetic signal, which can eliminate the interference of accidental factors effectively, and provide the variation pattern of the magnetic signal in different deformation stages. Results indicate that the magnetic memory signal has special sensitivity to the initial yielding stage of the material. The characteristic of this stage is that $\overline{\mathrm{dH} / \mathrm{dx}}$ experiences large fluctuation, whereas it does not necessarily get the maximum value. The mechanism of this phenomenon is that the non-uniform plastic strain changes the spontaneous magnetic field $\mathrm{H}_{\mathrm{p}}$ inside the metal. In the elastic stage, $\mathrm{H}_{\mathrm{p}}$ is linearly increasing with $\mathrm{x}$, where $\overline{\mathrm{dH} / \mathrm{dx}}$ is almost constant. In the strengthening stage with uniform large deformation, $\mathrm{H}_{\mathrm{p}}$ gradually becomes stable and tends to zero.
\end{abstract}

\section{Introduction}

Magnetic memory testing of metal material is a new type of non-destructive detection technology proposed by Russian scholar Dubov [1]. It is widely used in the early defect diagnosis of ferromagnetic material equipment. In order to apply this method more accurately in the engineering, lots of researches have been done by scholars across the world, which are focused on the relationship between magnetic signals and the material stress and strain. Tensile test is the most common research method used, since it has a uniform stress state and covers three typical deformation stages: the elastic stage, the yielding stage and the strengthening stage. Due to many influencing factors, the two basic parameters of magnetic signals (the magnetic field strength $\mathrm{H}_{\mathrm{p}}$ and the magnetic field strength derivative $\mathrm{dH} / \mathrm{dx}$ ) usually behave randomly [3]. Hence, in previous studies, $\mathrm{H}_{\mathrm{p}}$ and $\mathrm{dH} / \mathrm{dx}$ are mostly characterized qualitatively [2]. Therefore, what causes the magnetic signal changes and how to quantitatively characterize the signal have always been the key problem of the magnetic memory research.

A lot of studies have been conducted upon this problem. Xu et al [4], Sergey et al [5], and Duan et al [6] proposed that the magnetic field within the metal changes with the strain variation, while the non-uniform magnetic field is caused by nonhomogeneous deformation. Li et al [7,8] argued that the magnetic memory signal and the stress centralizing have a clear correspondence, which confirms feasibility of the detection method based on metal magnetic memory. Huang et al [9] and $\mathrm{Yu}$ et al [10] found that the magnetic memory is sensitive to the yielding process. It was also indicated that magnetic distortion is a direct characterization and unique phenomenon of local yielding. In summary, previous studies concentrated on phenomenon description and qualitative analysis, and therefore further research on the nature of magnetic memory variation based on quantitative characterization is in high demand.

In this paper, we focus on the key problem of magnetic signal characteristics in the strain varying 
processes of metal materials. Fundamental influencing factors of the two parameters in the magnetic memory test are explored, trying to give a quantitative characterization. By stretching experiments of a large specimen, the magnetic memory signal analysis is carried out in different stages. The local derivative method proposed in this paper is used to find out characteristics of the magnetic signal in each stage. Relationship between the magnetic field strength and its derivative is analyzed.

\section{Experiments}

In order to study relationship between the magnetic signal and the deformation stages of the material, the tensile test was carried out with Q345R steel. The magnetic memory test is conducted at the typical stages of the stretching process. The mechanism and influence factors of the magnetic signal are analyzed by dealing with the magnetic signals at each stage.

\section{Experiment equipment and specimen}

The Zwick/Roell Z100 and the Schenck RAS-250 electronic universal testing machines were used to carry out the tensile test. The Zwick/Roell Z100 testing machine can precisely control the strain of the specimen in the yield stage, which can provide accurate relationship between the strain and magnetic memory Signals. The Zwick/Roell Z100 test machine had insufficient range because of the high breaking load required for the test specimen, so the tests were carried on with the RAS-250 after the strengthening stage.

Magnetic memory measurement was conducted with the Russian-produced TSC-1M-4 type stress concentration detector, using a single channel sensor.

Q345R steel was used as the test material, and two specimens were used for the convenience of measurement. Considering the measurement accuracy and the rationality of the yield point capturing, specimens of a large size were applied, with the length as $350 \mathrm{~mm}$ and the thickness as $6 \mathrm{~mm}$, which is shown in Fig. 1. The applying of large specimens also effectively removes the magnetic signal interference caused by the clamping and the cross-section varying.

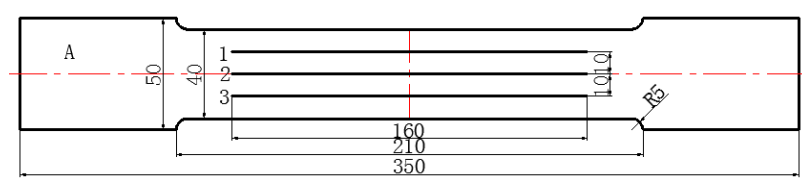

Figure 1. Specimen size and shape.

\section{Experiment Strategy}

Focusing on the factors affecting the magnetic signal, three stages of elasticity, yielding and strengthening are emphasized in the tensile process. Some studies have shown that the initial plastic deformation of the yielding stage is not uniform, thus the initial yield point on the specimen is random [7]. Magnetic memory method is a powerful means to accurately capture to the point. It is very important to quantitatively given the relationship between the strain and initial yield point, for the study on the mechanism of magnetic signal change.

In each test, the specimen is divided into two sides (A and B), and there are three detection lines $(1,2$, and 3$)$ on each side, providing a good detection direction, as shown in Fig. 1.

In order to eliminate the influence of the chuck on the magnetic signal of the test piece, the unloading method was adopted, which first removes the test piece for detection and then continue the loading. The magnetic memory signal detection was conducted for several times across the elastic, yielding, strengthening and necking stages of the tensile process. In the elastic stage, the selected stresses were $110 \mathrm{MPa}, 220 \mathrm{MPa}$ and $330 \mathrm{MPa}$. In the yield stage, 5 points were taken as the stop points for measurement. In the strengthening stage, 5-6 points are randomly selected as the measuring points. One or two points were measured in the necking stage, and the test piece was measured once after fracture.

In order to make the test results more accurate, it is needed to remove the impact of the earth magnetic field. TSC-1M-4 tester of stress concentration is capable of compensating the earth magnetic field. In the test, an area without electromagnetic interference was firstly selected as 
magnetic memory signal measurement location. The vacant magnetic field was measured several times for some zones in the location, and the one with the most stable vacant magnetic field is chosen as the test zone.

\section{Experiment Results}

\section{Process of stretching and signal measuring}

The stress-strain curve of the Q345R during the tensile magnetic memory test is shown in Fig. 2 and Fig. 3. Each point marked on the graph is the stop point for measurement. Each of the test pieces were measured 20 times.

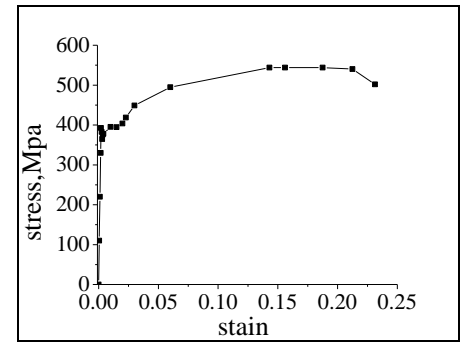

Figure 2. Strain-stress curve of $1 \#$ specimen

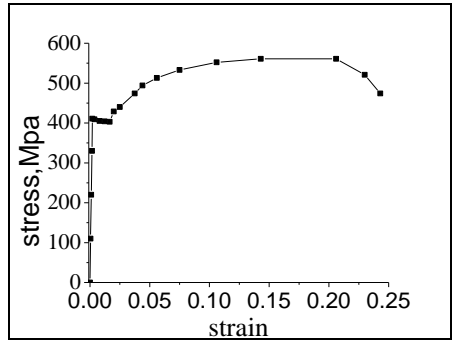

Figure 3. Strain-stress curve of 2\# specimen

\section{Magnetic memory detection results}

Magnetic memory test results of test piece No.1 are shown in Fig.4, where the abscissa is the measurement distance, and the vertical axis denotes the magnetic field strength $\mathrm{H}_{\mathrm{p}}$, with the unit as $\mathrm{A} / \mathrm{m}$.

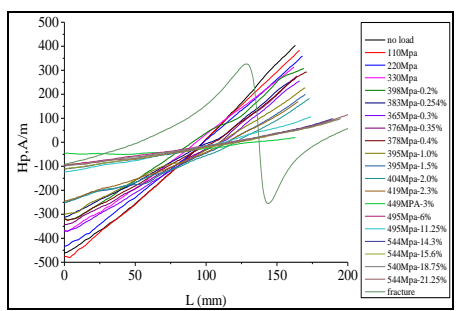

a)A1 Line

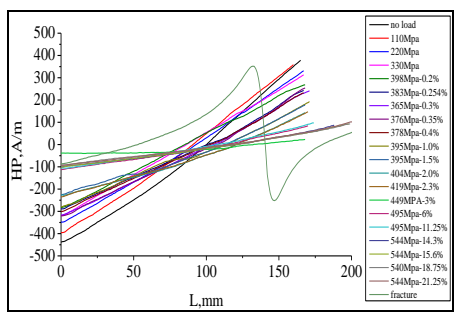

b)A2 Line

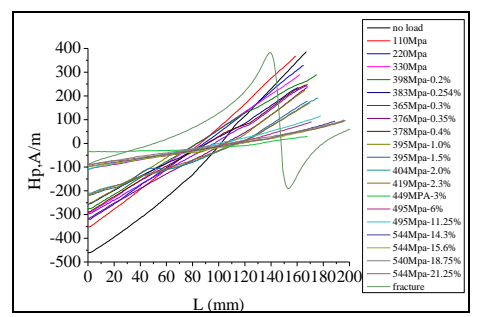

c)A3 Line

Figure 4. Magnetic memory test results of test piece No.1

\section{Analyses and Discussion}

\section{Data processing method}

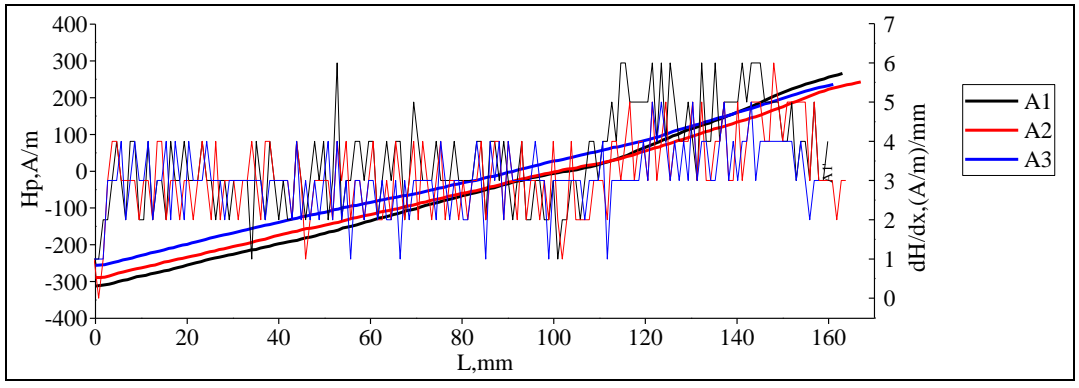

Figure 5. Primitive results of the magnetic memory measurement

Typical results of the magnetic memory measurement are shown in Fig. 5, which is the $\mathrm{H}_{\mathrm{p}}$ and $\mathrm{dH} / \mathrm{dx}$ curve in the initial yielding stage for three detection lines of Slide A of test piece No.2. It can be seen that only one line in the three $\mathrm{H}_{\mathrm{p}}$ curves bends slightly. The corresponding derivative curve has a local maximum value in the vicinity. However, the derivative curve also experiences some extreme values in other regions, so it is difficult to give a clear and accurate judgment on whether yielding happens and where is the yielding point. Therefore, it is necessary to deal with the original 
test data, so as to find an effective evaluation method.

The data processing principle proposed in this paper is as follows. (1) Normalization is conducted upon the $\mathrm{dH} / \mathrm{dx}$ values by calculating the mean local derivative $\overline{\mathrm{dH} / \mathrm{dx}}$ of a certain region. (2) The standard deviation of $\mathrm{dH} / \mathrm{dx}$ is obtained to evaluate the degree of dispersion. (3) The variation trend of the magnetic field and its derivative is analyzed based on the mean local derivative $\overline{\mathrm{dH} / \mathrm{dx}}$ and the standard deviation. Accordingly, the characteristics of the magnetic signal can be more clearly examined during all stages of the test piece deformation.

The magnetic signal data at the beginning and the ending sections of $5 \mathrm{~cm}$ length are removed, in order to make the measurement result more accurate. In account for the ease of analysis, the data is divided into 10 sections according to the detection distance. The mean local derivative can be calculated for each section along the testing line as follows,

$$
\overline{d H_{i} / d x}=\frac{1}{n} \sum_{j=1}^{n} \frac{d H_{j}}{d x}
$$

where $\overline{\mathrm{dH}_{\mathrm{i}} / \mathrm{dx}}$ is the local average value of $\mathrm{dH} / \mathrm{dx}$ in the $i$-th section, while $\mathrm{d} H_{j} / \mathrm{d} x$ is the derivative of the magnetic field at the $\mathrm{j}$-th point in the $\mathrm{i}$-th section. The standard deviation of $\mathrm{dH} / \mathrm{dx}$ can be calculated as,

$$
\sigma=\sqrt{\frac{1}{N} \sum_{i=1}^{n}\left(\overline{d H_{i} / d x}-\mu\right)^{2}}
$$

where $\sigma$ is the standard deviation of $\overline{\mathrm{dH} / \mathrm{dx}}$ across the whole test line, and $\mu$ is the overall average of $\overline{\mathrm{dH} / \mathrm{dx}}$. The overall standard deviation $\sigma$ represents the fluctuation of the mean local derivative. Meanwhile, the variation of $\overline{\mathrm{dH} / \mathrm{dx}}$ indicates that the magnetic field strength of the specimen is abruptly changed, which denotes a non-uniform deformation. The division of the detection line is shown in Fig. 6.

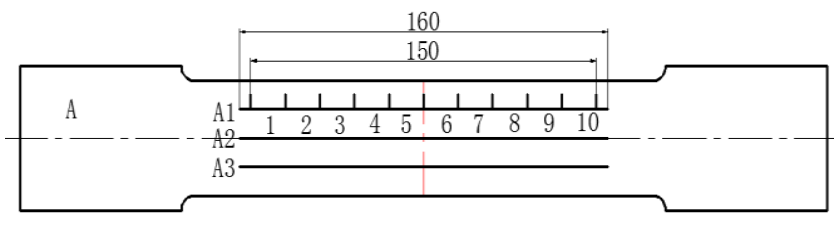

Figure 6. Division of the detection line

\section{Results after processing}

In the experiment, two specimens were tested under 20 different loading conditions. There were 6 detection lines on the A and B sides of each specimen, with 20 magnetic memory derivative data on each test line. According to the above principle, the data were processed and statistically analyzed, which are also separated into three groups corresponding to the three stages of the tensile process. The typical results are shown in Fig. 7.

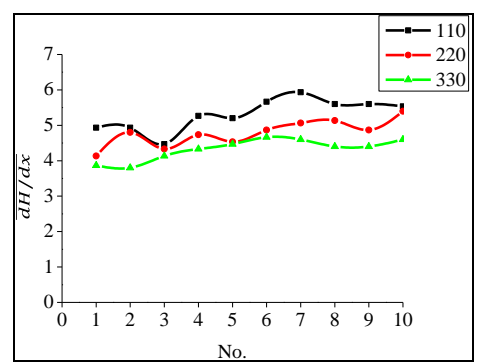

a) Elastic stage

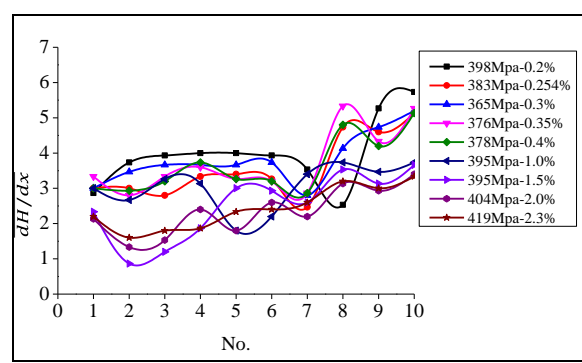

b) Yielding stage

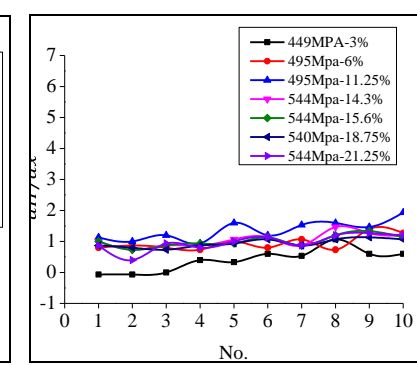

c) Strengthening stage

Figure 7. Mean local derivation of test piece No.1 in all stages 


\section{Magnetic signal distribution regulation}

(1) The original state

Results of $\mathrm{H}_{\mathrm{p}}$ and $\mathrm{dH} / \mathrm{dx}$ measurements at the original state without load on the A-plane of two specimens are shown in Fig. 8a. The mean local derivative $\overline{\mathrm{dH} / \mathrm{dx}}$ of each section is shown in Fig. 8b. It can be seen from Fig. 8 that the $\mathrm{H}_{\mathrm{p}}$ and $\mathrm{dH} / \mathrm{dx}$ of the two specimens are completely different, which indicates that the magnetic field $\mathrm{H}_{\mathrm{p}}$ of the specimen is non-uniform and random at the original state.

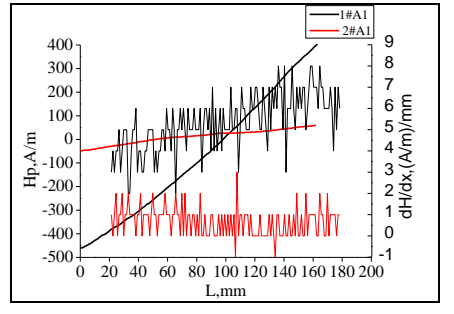

a)Primitive results

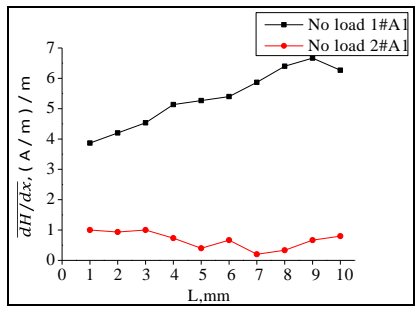

b) Mean local derivative

Figure 8. Magnetic memory signal without any load

(2) Elastic stage

Fig. 9a shows the result of the magnetic memory signal in the elastic stage. It can be seen that the magnetic field intensity is approximately linearly increasing with the distance, with the slope decreases as the stress increases. Fig. 7a shows the mean load derivative $\overline{\mathrm{dH} / \mathrm{dx}}$ at three different loads in the elastic stage, indicating that under the same load, $\overline{\mathrm{dH} / \mathrm{dx}}$ seems to be constant and does not change with the distance.

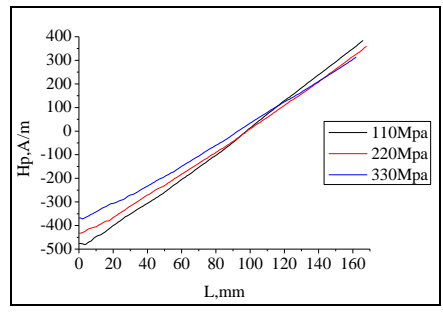

a) Primitive results

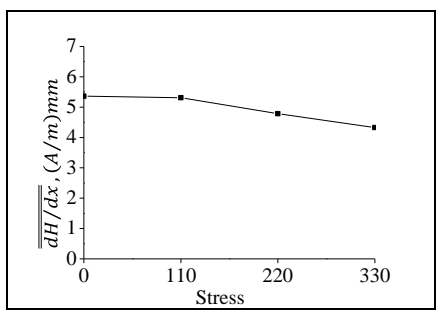

b) Overall average

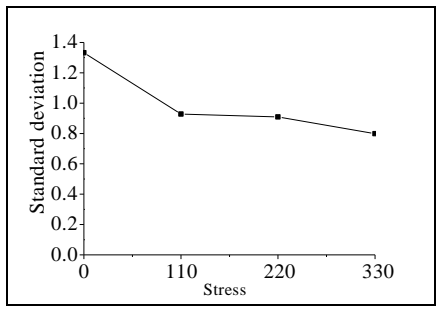

c) Overall Standard deviation

Figure 9. Results with different loading at the elastic stage

The overall average $u$ and the overall standard deviation $\sigma$ are calculated for the elastic stage, with the result shown in Figs. 9b and 9c. It can be seen from Fig. 9b that as the loading stress increases, the overall average $u$ decreases, consistent with the trend of Fig. 9a. The overall standard deviation of $\overline{\mathrm{dH} / \mathrm{dx}}$ shows to decrease gradually with the increase of the loading stress, indicating that the magnetic field of the specimen becomes more uniform after the stress is applied. In summary, we can induce that (1) the applied stress makes the magnetic field uniform, and that $\mathrm{dH} / \mathrm{dx}$ decreases with the increase of loading stress.

(3) The yielding stage

The $\mathrm{H}_{\mathrm{p}}$ and $\overline{\mathrm{dH} / \mathrm{dx}}$ curves of the yielding stage are shown in Fig. 10. It is shown in Fig.10a that a local shift of the $\mathrm{H}_{\mathrm{p}}$ curve occurs in the yielding stage. Fig.10b also shows that the mean derivative experiences a local deviation, where $\overline{\mathrm{dH} / \mathrm{dx}}$ has a significant decrease up to unit. Combining figures from Fig.10b to Fig.10e, we can induce that the local deviation is moving from right to left during the whole yielding stage (position 8 for $0.2 \%$ strain and position 2 for $1.5 \%$ strain). It proves that initial yielding happens at a certain cross-section. Referring backward to Fig.5, we can find only one line of the three has a local shift, indicating that the initial yielding even takes place at one particular point on the certain cross-section. It further proves that the yielding happens randomly. Fig.10f shows the case of fully yielding, where $\overline{\mathrm{dH} / \mathrm{dx}}$ decreases and tends to stable. 
In the tensile process, the stress state and stress value keep same in all cross-sections of the specimen from the macroscopic point of view. However, from the microscopic point of view, there is inhomogeneous plastic strain in the initial yielding stage [11]. The magnetic memory test applies just to capture this local change. The results also show that the non-uniform plastic deformation occurs between $0.2 \%$ and $2.3 \%$ strain during the yielding process.

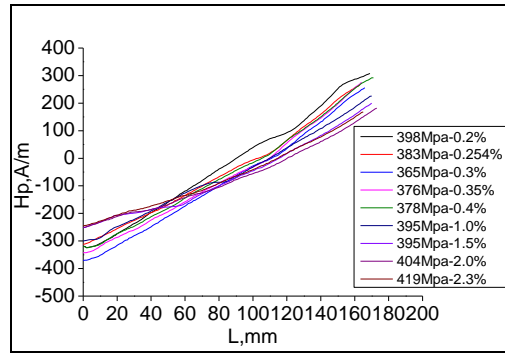

a) Magnetic field of A1, yielding

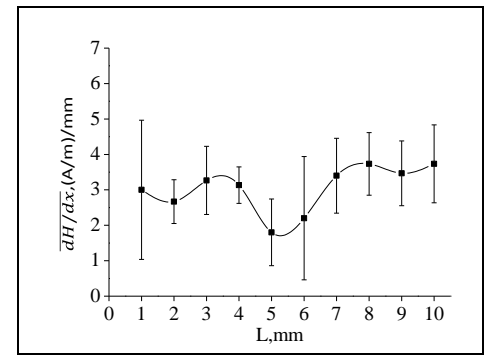

d) yielding with $1.0 \%$ strain

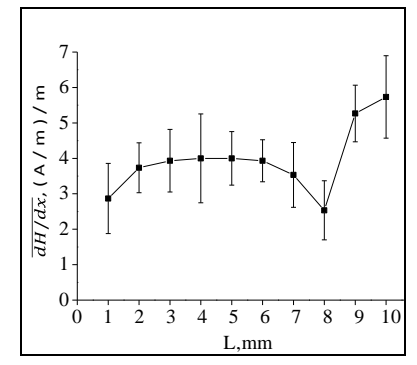

b) yielding with $0.2 \%$ strain

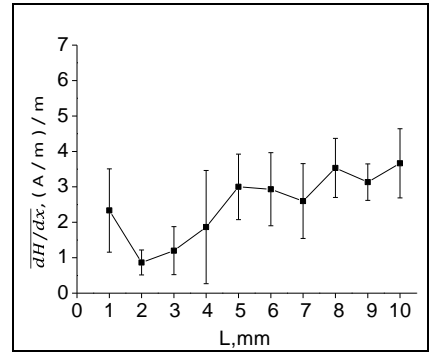

e) yielding with $1.5 \%$ strain

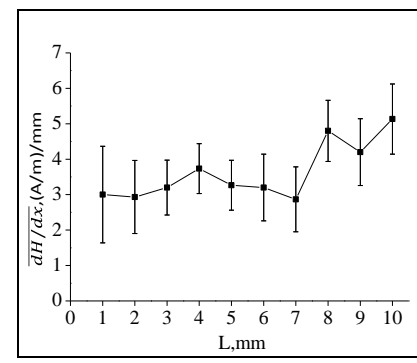

c) yielding with $0.4 \%$ strain

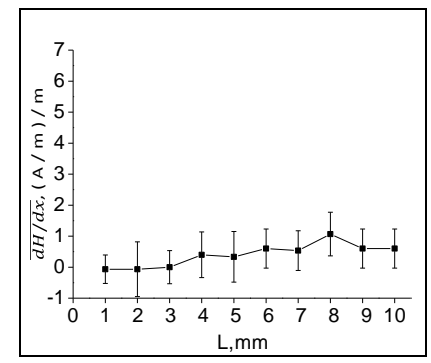

f) strengthening with $3 \%$ strain

Figure 10. Moving of the local deviation at the yielding stage

(4) The strengthening stage

It can be seen from Fig.7c that in the strengthening stage, the mean local derivative of the specimen gradually becomes uniform with the stress increases, while $\overline{\mathrm{dH} / \mathrm{dx}}$ is gradually smaller, varying from zero to two. It indicates that the $\mathrm{H}_{\mathrm{p}}$ curve tends to be horizontal when large deformation occurs.

\section{Overall standard deviation in all stages}

The value of the overall standard deviation $\sigma$ reflects the degree of $\overline{\mathrm{dH} / \mathrm{dx}}$ variation. The overall standard deviation of 20 tests during the whole experiment is shown in Fig.11. It can be seen that $\sigma$ is 1.33 without any load, $\sigma$ is smaller than 0.93 at the elastic stage, $\sigma$ varies between 1.18 and 1.30 after entering the yielding stage, and $\sigma$ becomes smaller than 0.87 after the strengthening stage. It is clear from the results that $\overline{\mathrm{dH} / \mathrm{dx}}$ only changes considerably in the yielding stage, that is, when the initial yielding happens. The shape of $\mathrm{H}_{\mathrm{p}}$ curve changes and $\mathrm{dH} / \mathrm{dx}$ shows a large mutation.

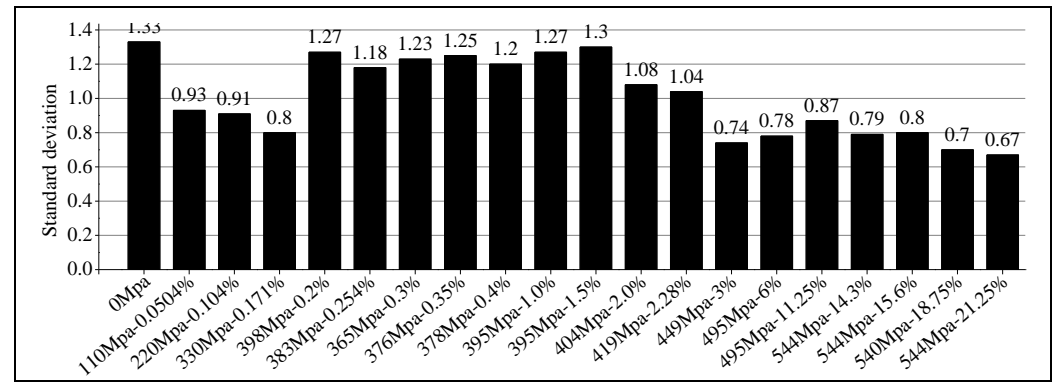

Figure 11. The overall standard deviation in the tensile process

\section{Conclusion}

(1) The local derivative mean method proposed in this paper can effectively eliminate the interference of accidental factors. It can be used to evaluate the variation trend of $\mathrm{H}_{\mathrm{p}}$ and $\mathrm{dH} / \mathrm{dx}$ 
objectively. The variation law of the magnetic signal in each deformation stage can be analyzed with the method.

(2) The magnetic memory test is particularly capable for detection of the initial yielding. An important characteristic of the magnetic signal at this stage is that the mean local derivative $\overline{\mathrm{dH} / \mathrm{dx}}$ has a large deviation rather than a maximum value. The non-uniform plastic deformation causes $\overline{\mathrm{dH} / \mathrm{dx}}$ to change locally, and the changing point moves as the strain increases. The mechanism is that non-uniform plastic deformation of ferromagnetic metal causes its internal spontaneous magnetic field $\mathrm{H}_{\mathrm{p}}$ to change.

(3) The magnetic field strength $H_{p}$ exhibits randomness when the metal material is not subjected to any load. The spontaneous magnetic field in the elastic phase is homogenized, and the $\mathrm{H}_{\mathrm{p}}$ curve presents to be a linear line, with $\overline{\mathrm{dH} / \mathrm{dx}}$ behaving almost as a constant. Homogeneous plastic deformation takes place in the strengthening stage. As the plastic deformation increases, the $\mathrm{H}_{\mathrm{p}}$ gradually becomes homogeneous, and the $\overline{\mathrm{dH} / \mathrm{dx}}$ becomes smaller and tends to zero.

\section{References}

[1] Dubov A.A. Diagnostics of boiler tubes with usage of metal magnetic memory [M]. Moscow: Energoatomizdat, 1995. 112p.

[2] Hao Su, Ming Chen. Experimental and theoretical analysis of metal magnetic memory signals in the stress concentration area of 45\# steel under tensile testing[J].International Journal of Applied Electromagnetics and Mechanics, 46 (2014), 271-280

[3] Haihong Huang, Cheng Yang, Zhengchun Qian. Magnetic memory signals variation induced by applied magnetic field and static tensile stress in ferromagnetic steel [J]. Journal of Magnetism and Magnetic Materials, 416 (2016) , 213-219.

[4] Mingxiu $\mathrm{Xu}$, Zhanghua Chen, Minqiang Xu. Micro-mechanism of metal magnetic memory signal variation during fatigue [J]. International Journal of Minerals, Metallurgy and Materials, 2014, 21(3):259-264.

[5] Kolokolnikov Sergey, Dubov Anatoly, Steklov Oleg. Assessment of welded joints stress-strain state inhomogeneity before and after post weld heat treatment based on the metal magnetic memory method [J]. Welding in the World, 2016, 60(4):665-672.

[6] Zhenxia Duan, et al. Magnetizing reversal characteristic of $40 \mathrm{Cr}$ steel during stress-magnetizing process [J]. Journal of Iron and Steel Research, 2016, 21(01):77-80.

[7] Ji-Min L I, Zhang Y L. Study of Magnetic Memory Effect of Q235 Steel Material under Tension Condition[J]. Pressure Vessel Technology, 2008. (07):10-22.

[8] Ji-Min L I, Zhang Y L, Shen G T. Study on Magnetic Memory Effect in Tensile Process and Micro-microstructure[J]. Pressure Vessel Technology, 2009. (08):15-20.

[9] Haihong Huang, Shilin Jiang, Rujun Liu. Investigation of Magnetic Memory Signals Induced by Dynamic Bending Load in Fatigue Crack Propagation Process of Structural Steel [J]. J Nondestruct Eval, 2014, 33:407-412.

[10] Min Yu, et al. Research on New Test Method for Local Yield Based on Metal Magnetic Memory[J]. Hot Working Technology, 2014. 43(17):87-92

[11] Min Yu, et al. Research on Yield Effect of Metal Magnetic Memory Signals of 20g Low Carbon Steel[J]. Hot Working Technology, 2015. 44(02):69-72. 